\title{
Les verbes transitifs de localisation statique Diathèses, modes d'action et sélection lexicale
}

\author{
Denis Le Pesant \\ Université Paris 10 Nanterre \\ MoDyCo (CNRS \& Université Paris 10) \\ denis.lepesant@wanadoo.fr
}

\section{Introduction}

L'acte de langage consistant à localiser un objet, c'est-à-dire déterminer la relation qu'il entretient avec un autre objet servant de point de repère, est réalisé par l'emploi, par le locuteur, des prédicats de localisation. La localisation peut être interne ou externe et être spécifiée selon beaucoup de paramètres, tels la présence ou l'absence de contact, l'antériorité ou la postériorité par rapport au locuteur, la distance etc. (cf. entre autres C. Vandeloise 1986, A. Borillo 1998 et M. Aurnague 2004). Les prédicats de localisation sont des prédicats relationnels à deux arguments : l'argument thème (de la localisation) et l'argument repère (de la localisation). Nous appelons prédicats de localisation statique (par opposition aux prédicats de changement de localisation) ceux qui projettent sur l'argument thème l'information selon laquelle l'objet qu'il désigne est statique ; c'est le cas par exemple de la préposition en-haut de, du verbe transitif direct occuper, et des verbes à argument prépositionnel locatif résider à et tomber de (ce dernier n'étant un verbe de localisation statique que si son mode d'action est statif, et non pas télique).

Ce texte est un essai de classification syntactico-sémantique des verbes transitifs de localisation statique selon les critères de la forme de leurs diathèses et de leur sélection lexicale. Les propriétés syntaxiques de permutation d'arguments et de changement du nombre des arguments sont insuffisantes à définir de façon complète les diathèses verbales. Il faut y adjoindre les propriétés de mode d'action des verbes, ainsi que celles de linéarisation des arguments, c'est-à-dire l'ordre dans lequel se trouvent les arguments thème et repère. L'analyse met en évidence l'existence de quatre catégories de verbes transitifs directs ou indirects de localisation statique : les types côtoyer, précéder, parsemer, occuper, les types contenir, regorger de, les types bourrer de, gorger de, et les types qui combinent des diathèses à argument complément d'objet direct ou indirect avec des diathèses à argument locatif (inclure, abonder, semer, charger, baigner, envelopper $)^{1}$.

\section{$2 \quad$ Les critères de la classification}

\subsection{Notions de diathèse et de schéma de diathèses}

Sous le terme de diathèse, on désigne l'association de deux variantes de forme de la structure argumentale d'un verbe ou d'une classe de verbes. Une diathèse résulte soit d'une permutation des arguments (diathèses de permutation), soit d'une augmentation ou d'une diminution du nombre des arguments (diathèses de réduction ou d'augmentation). Certaines diathèses associent les deux opérations, telle la diathèse de neutralité (cf. Boons, Guillet \& Leclère 1976), que B. Levin 1993 appelle Causative Alternation: le cuisinier a cuit le rôti vs le rôti a cuit. L'aspect morphologique est complémentaire de l'aspect syntaxique : certaines diathèses se manifestent en effet par un changement dans la conjugaison. Par exemple, le verbe passe de la voix active à la voix passive ou inversement; ou bien il passe de la voix moyenne (dite aussi pronominale) à la voix active ou inversement, comme dans : le train s'est arrêté vs on a arrêté le train. Toutes les opérations morphosyntaxiques sous-jacentes aux diathèses correspondent à ce qu'on appelle traditionnellement des transformations. Pour conserver à la diathèse sa propriété d'être un couple de variantes syntaxiques, il faut envisager l'éventualité qu'une même variante puisse appartenir à 
deux diathèses distinctes. Enfin, une même forme syntaxique ayant la possibilité d'avoir deux modes d'action différents sera dédoublée en deux variantes distinctes, comme nous allons le voir dans la Section 2.2 .

Nous appelons schéma de diathèses l'ensemble des éventuelles diathèses caractéristiques d'un verbe ou d'une classe de verbes. Un schéma de diathèses peut avoir plus d'une ou deux diathèses : par exemple, celui des verbes du type parsemer en compte quatre (cf. infra Section 3.3). Enfin, un verbe, même transitif, peut évidemment se caractériser par le fait de n'avoir pas de diathèses (cf. par exemple infra Section 4).

\subsection{Les variations de mode d'action dans les schémas de diathèses}

La prise en compte des variations de mode d'action qui se manifestent au sein d'un schéma de diathèses permet d'enrichir considérablement la définition des classes syntactico-sémantiques de verbes.

Les quatre modes d'action (Aktionsart) ont été initialement conçus par Z. Vendler 1967 comme un système de classification sémantique des verbes, distribués en états, activités (= processus non téliques), accomplissements (= processus téliques, ou réalisations non ponctuelles) et réalisations ponctuelles (= les achievements de Vendler). Le meilleur usage qu'on peut faire de ces distinctions, c'est de les appliquer aux différentes variantes qui se manifestent au sein d'un schéma de diathèses, plutôt qu'à l'entrée verbale ellemême.

La définition des modes d'action est fondée sur des propriétés aspectuelles, par exemple les auxiliaires aspectuels autorisés par le verbe, les choix à faire au sein des dichotomies perfectif-imperfectif et inaccompli-accompli. Par ricochet, les propriétés temporelles entrent aussi en ligne de compte ; ainsi, l'aspect perfectif exclut le temps présent. Les verbes d'état se caractérisent par le fait de refuser l'auxiliaire aspectuel de progressivité être en train de ; de ne pas pouvoir être sous la portée de la préposition temporelle en; d'accepter d'être sous la portée de la préposition pendant à condition d'être conjugués à l'aspect perfectif inaccompli et aux temps passé ou futur; d'accepter enfin d'être sous la portée de depuis à condition d'être conjugués à l'aspect imperfectif inaccompli et aux temps présent et passé. En revanche, les verbes d'accomplissement acceptent l'auxiliaire de progressivité être en train de ; peuvent être sous la portée de la préposition temporelle en, à condition d'être conjugués à l'aspect perfectif inaccompli aux temps passé et futur ; acceptent enfin d'être sous la portée de depuis, à condition d'être conjugués à l'aspect imperfectif inaccompli aux temps présent et passé.

Dans l'analyse des classes de verbes, associer les modes d'action aux propriétés syntaxiques équivaut à faire intervenir un nombre considérable de critères supplémentaires non seulement sémantiques, mais aussi syntaxiques. En effet, la relation des verbes avec quelques prépositions temporelles cruciales ( $a$, pendant, en, depuis, pour) n'est rien d'autre qu'une relation de sélection lexicale opérée par les prépositions temporelles, qui sont des prédicats.

Les propriétés de mode d'action mettent en évidence la nécessité de dédoubler parfois une même forme syntaxique en deux variantes du schéma de diathèses, comme dans les exemples (1) et (2) :

(1)a (Pendant deux minutes), la neige couvrit le sol

(1)b (En deux minutes), la neige couvrit le sol

(2)a (Pendant deux minutes), le sol fut couvert de neige

(2) $\mathrm{b} \quad$ (En deux minutes), le sol fut couvert de neige

Dans chacun de ces couples d'exemples, les phrases diffèrent par le mode d'action. Nous devons donc associer aux tiroirs de voix des spécifications de mode d'action : la variante (1)a est à la voix active stative, tandis que la variante (1)b est à la voix active télique non ponctuelle; la variante (2)a est à la voix passive stative, tandis que la variante (2)b est à la voix passive télique. 


\title{
2.3 Le problème des phrases à argument causatif ou agentif
}

Nous avons évoqué plus haut les notions de diathèse d'augmentation ou de réduction du nombre des arguments au sein d'un schéma de diathèses. Nous sommes convaincu que la prise en compte des propriétés sémantiques de chaque variante nous oblige à admettre que les deux variantes d'une diathèse peuvent être orientées, et qu'elles peuvent en particulier être orientées dans le sens du passage d'une forme courte à une forme longue.

Selon une opinion fort répandue, les structures non causatives statives (dites aussi, de façon caractéristique, résultatives) au sein d'un même schéma de diathèses sont dérivées d'une structure causative, plus longue d'un argument (l'argument agent ou l'argument cause). Tout au moins, se plaçant du point de vue plus strictement sémantique, certains présentent-ils les structures statives de localisation comme décrivant nécessairement l'état résultant de l'acte de mettre quelque chose dans ou sur quelque chose, telle B. Levin 1993:120, à propos des Fill Verbs.

Cette vision des choses s'explique surtout par le fait que la notion d'effacement d'argument passe pour être plus élégante que celle d'ajout d'argument, parce qu'elle permet la récupération des segments effacés dans le discours ou dans le contexte extralinguistique. Toutefois, dans le cas qui nous occupe, elle est justement mise en échec par l'impossibilité fréquente de récupérer l'argument prétendument effacé, sauf à invoquer des agents métaphysiques ou à personnifier les phénomènes. Soient les phrases passives statives suivantes :

\author{
Les pôles sont recouverts de glaces \\ Ce soir, le ciel est constellé d'étoiles \\ Il y a à $1000 \mathrm{~m}$ sous terre une couche sablonneuse qui est saturée d'hydrocarbures \\ En l'espace de 10 ans, la ville s'est entourée d'une zone industrielle désordonnée \\ Dans cette région, les buttes sont parfois coiffées de fermes fortifiées \\ Les plaines de cette région se sont ponctuées de bosquets au fil des siècles
}

Il est impossible de dériver ces phrases de prétendues sources à argument causatif ou agentif, tout autant qu'il serait fâcheux de dériver «cet objet est jaune » de «quelque chose ou quelqu'un a jauni cet objet ». Du point de vue purement sémantique, il est inenvisageable de les présenter comme des résultats d'accomplissements. Ce phénomène de "préséance» possible des variantes statives par rapport aux variantes causatives dans le vocabulaire des verbes transitifs de localisation statique sera pris en compte dans notre travail de classification.

\subsection{Sur un type particulier de voix médio-passive}

Un type particulier de voix médio-passive se manifeste dans certains schémas de diathèse de la catégorie de verbes transitifs de localisation. Voici quelques exemples de diathèse de permutation faisant alterner la voix active télique avec la voix médio-passive télique :

En dix ans de temps, une épaisse forêt a recouvert ce sol aride

En dix ans de temps, ce sol aride s'est recouvert d'une épaisse forêt

En 10 minutes, un redoutable verglas nappa les trottoirs

En 10 minutes, les trottoirs se nappèrent d'un redoutable verglas

En l'espace de 20 ans, les caténaires de TGV ont jalonné la France dans tous les sens En l'espace de 20 ans, la France s'est jalonnée de caténaires de TGV dans tous les sens

En 9 ans, deux clochers flanquèrent la nef de l'église, et des murs épais entourèrent la ville En 9 ans, la nef de l'église se flanqua de deux clochers, et la ville s'entoura de murs épais

Quand le mode d'action du verbe est l'accomplissement, la valeur sémantique de la voix pronominale moyenne est celle du devenir. On peut s'interroger sur cette notion. Est-elle d'essence aspectuelle? Nous 
le croyons dans la mesure où l'expression « devenir telle ou telle chose » est paraphrasable en «cesser de ne pas être telle ou telle chose», laquelle met en jeu un aspectuel terminatif. En associant, dans certains schémas de diathèses, la structure à voix pronominale avec une structure à voix active, nous supposons que la variante pronominale est plus particulièrement de nature médio-passive. Par exemple, la phrase «en 10 minutes, les trottoirs se nappèrent d'un redoutable verglas» est présentée comme le correspondant médio-passif de « en 10 minutes, un redoutable verglas nappa les trottoirs ».

\subsection{Critères généraux de classification des verbes transitifs de localisation statique}

Nous répartissons les verbes de localisation statique en quatre classes en prenant comme premier critère la forme de la variante ou des variantes qui sont statives. Le verbe dans sa variante stative peut être conjugué à la voix active (Le sang imprègne la compresse) ou à la voix passive (La compresse est gorgée de sang). Parfois, il existe deux variantes statives dans le même schéma de diathèses (Le sang imprègne la compresse vs La compresse est imprégnée de sang). Par convention arbitraire, nous avons privilégié la variante stative active, qui est prioritaire dans la définition d'une classe ; c'est le cas du verbe imprégner, qui est rangé dans les verbes à voix active stative, bien qu'il dispose aussi de la voix passive stative.

Le deuxième critère de classification concerne la position respective de l'argument thème et de l'argument repère des verbes transitifs de localisation statique. Dans des verbes à la voix active stative tels que contenir et abriter, le SN en position sujet joue le rôle de repère de la localisation. En revanche, les verbes occuper et imprégner sous-catégorisent un argument sujet thème de la localisation. La prise en compte des types de schémas de diathèses éventuels et de la sélection lexicale permet de subdiviser chacune des quatre catégories générales en un certain nombre de sous-types. On obtient par le jeu conjoint des différents critères la classification suivante :

- Verbes de relation thème-repère à voix active stative (Section 3): sous-types côtoyer, précéder, parsemer, occuper

- Verbes de relation repère-thème à voix active stative sans diathèses (Section 4) : sous-types contenir, regorger de

- Verbes de relation repère-thème à voix passive stative sans correspondant actif (Section 5) : soustypes être bourré de, être gorgé de

- Verbes combinant les diathèses transitives directes ou indirectes, et les diathèses à argument prépositionnel locatif (Section 6) : sous-types inclure, abonder en, être semé de, être chargé de, baigner.

\section{Les verbes de relation thème-repère à voix active stative (sous- types côtoyer, précéder, parsemer, occuper)}

\subsection{Sous-type côtoyer : voix active stative non passivable}

Les verbes transitifs directs du sous-type côtoyer étant non passivables, ils n'ont pas de diathèses.

$$
\text { Ma maison jouxte celle de François vs * La maison de François est jouxtée par la mienne }
$$

Les verbes côtoyer et avoisiner sont des parasynonymes de être près de (localisation externe sans contact avec proximité). Les verbes toucher et jouxter sont des parasynonymes de être tout contre (localisation externe avec contact) et le verbe regarder est un parasynonymes de être en face de (localisation externe sans contact sur l'axe frontal, pôle antérieur). 


\subsection{Sous-type précéder/suivre : voix active stative \& voix passive stative en de}

Les verbes précéder et suivre se caractérisent par le schéma de diathèses suivant :

(9) Diathèse de permutation faisant alterner la voix active non causative stative avec la voix passive non causative stative. Dans la variante passive, le complément du verbe est introduit par la préposition de.

Pendant deux ans, un jardinet a précédé ma maison vs ma maison a été précédée d'un jardinet

Depuis deux ans, un jardinet précédait ma maison vs ma maison était précédée d'un jardinet

Les verbes précéder et suivre sont des parasynonymes respectivement de être devant et être derrière (localisation externe avec ou sans contact sur l'axe frontal ; les pôles sont, respectivement, l'antérieur et le postérieur).

\subsection{Sous-type parsemer : voix active stative, voix passive stative en de, voix moyenne \& causatif (voix active et passive téliques)}

La diathèse de permutation faisant alterner la voix active stative avec la voix passive stative en de (ex. des pièces d'or remplissent ce sac vs ce sac est rempli de pièces d'or) correspond approximativement à celle de l'anglais que B. Levin 1993 appelle Locatum Subject Alternation, avec un complément prépositionnel en with. Levin critique l'opinion selon laquelle ce complément serait un instrumental. Ce ne saurait de toute façon être le cas en français, car, outre le fait que la valeur de de est plutôt causale qu'instrumentale, le complément introduit par cette préposition est presque toujours obligatoire dans la diathèse $(*$ la cour est parsemée, * la colline est coiffée, * le sol est couvert), ce qui ne serait pas le cas si le complément était instrumental. On a plutôt affaire ici à une variété particulière de passivation impliquant la préposition de. La passivation en de se manifeste du reste dans d'autres vocabulaires (ex. être apprécié, aimé de/par tout le monde) $)^{2}$.

Les verbes du sous-type parsemer se caractérisent par le schéma de diathèses suivant :

(10)-1 Diathèse de permutation faisant alterner la voix active non causative stative avec la voix passive non causative stative. Dans la variante passive, le complément du verbe est introduit par la préposition $d e$, et il est obligatoire pour la quasi-totalité des verbes de la classe.

Pendant deux jours, les feuilles mortes (parsemèrent, parsèmeront) la cour

Depuis deux jours, les feuilles mortes (parsèment, parsemaient) la cour

Pendant deux jours, la cour (fut, sera) parsemée de feuilles mortes

Depuis deux jours, la cour (est, était) parsemée de feuilles mortes

(10)-2 Diathèse de permutation faisant alterner la voix active non causative télique avec la voix médiopassive télique

En deux minutes, les feuilles mortes (parsemèrent, parsèmeront) la cour

Depuis deux minutes, les feuilles mortes (parsèment, parsemaient) la cour

En deux minutes, la cour (se parsema, se parsèmera) de feuilles mortes

Depuis deux minutes, la cour (se parsème, se parsemait) de feuilles mortes

(10)-3 Diathèse de permutation faisant alterner la voix active causative télique non ponctuelle (accomplishment) avec la voix passive télique. 
En deux minutes, le vent (parsema, parsèmera) la cour de feuilles mortes

Depuis deux minutes, le vent (parsème, parsemait) la cour de feuilles mortes

En deux minutes, la cour (fut, sera) parsemée de feuilles mortes par le vent

Depuis deux minutes, la cour (est, était) parsemée de feuilles mortes par le vent

(10)-4 Réduction de la variante causative passive télique (10)-3 :

En deux minutes, la cour (fut, sera) parsemée de feuilles mortes

Depuis deux minutes, la cour (est, était) parsemée de feuilles mortes

La plupart des verbes de cette catégorie sont de localisation holistique.

\section{- Verbes de localisation interne}

Les verbes embarrasser et encombrer localisent un concret solide à l'intérieur d'un objet creux. Les verbes remplir et emplir se distinguent des précédents par le fait que l'objet thème peut être un concret massif. Quant aux verbes imbiber, mouiller, tremper, imprégner et saturer, ils expriment, avec des nuances diverses d'intensité, la localisation d'un liquide à l'intérieur d'un concret absorbant. Les verbes peupler et infester localisent (le second avec une nuance très péjorative) des êtres animés dans ou sur un objet concret, ou dans un lieu.

\section{- Verbes de localisation externe avec contact (parasynonymie avec la préposition sur)}

Les verbes couvrir, recouvrir, tapisser et revêtir peuvent être considérés comme les hyperonymes des verbes de cette sous-catégorie qui expriment une localisation holistique. Le verbe enrober sélectionne en position d'argument repère des noms d'objets tridimensionnels. Les verbes consteller, joncher et parsemer expriment le fait que l'objet en position d'argument thème est discontinu ; de fait, les noms qu'ils sélectionnent sont obligatoirement au pluriel. Les verbes jalonner et ponctuer sont proches des précédents ; ils en diffèrent par le fait qu'ils spécifient que les objets en position d'argument thème sont alignés. Le verbe hérisser sélectionne aussi des noms pluriels ; il spécifie que les objets désignés sont verticaux. Les verbes coiffer, couronner et surmonter localisent un objet vertical par rapport à un objet repère lui-même vertical, mais sans effet holistique nécessaire.

Le verbe inonder ne semble pas accepter la voix médio-passive. D'autre part, la variante passive stative peut réduire son complément, quand c'est un nom de liquide qui est sélectionné dans cette position : la plaine est inondée.

\section{- Autres verbes de localisation externe avec ou sans contact (parasynonymie avec les prépositions le long de, autour de, à côté de et de part et d'autre de)}

Les verbes border, franger et ourler localisent, comme le fait la préposition le long de, un objet allongé par rapport à un autre objet allongé. Les verbes ceinturer, entourer et environner localisent des objets discrets ou massifs comme le fait la préposition autour de. Quant à la localisation opérée par le verbe flanquer, elle est proche de celle qu'opèrent les prépositions à côté de ou de part et d'autre de (ex. L'église est flanquée d'un campanile; l'église est flanquée de deux maisons anciennes).

\subsection{Sous-type occuper : voix active stative \& voix passive stative en par}

Les verbes du sous-type occuper se caractérisent par le schéma de diathèses suivant :

(11) Diathèse de permutation faisant alterner la voix active non causative stative avec la voix passive stative. Dans la variante passive, le complément du verbe est introduit par la préposition par. Il 
est obligatoire, sauf pour quelques verbes à sujet humain.

Une énorme armoire occupe la moitié de la chambre

La moitié de la chambre est occupée par une énorme armoire

\section{- Verbes de localisation interne}

Les verbes accaparer, envahir et occuper 1 ainsi que, dans un registre plus ou moins familier, les verbes bouffer, dévorer et manger réalisent une localisation interne ou externe avec contact, avec effet holistique. Les verbes habiter, occuper 2 et squatter se distinguent des précédents par le fait qu'ils sélectionnent des noms humains en position d'argument thème, et des noms de logements en position d'argument repère ainsi que par leur effet non nécessairement holistique; de plus, ils se caractérisent par le fait d'avoir une deuxième diathèse, puisqu'ils peuvent sous-entendre le complément en par (ex. Ce logement est squatté).

\section{- Verbes de localisation externe}

Le verbe submerger effectue une localisation externe holistique avec contact des liquides par rapport à une surface horizontale. Les verbes cerner et encadrer sont à peu près synonymes, respectivement, des prépositions être autour et être de part et d'autre de. Quant aux verbes dominer et surplomber, ce sont des verbes de localisation externe sans contact sur l'axe vertical, pôle supérieur ; ce sont des parasynonymes de la préposition être au-dessus de.

\section{Les verbes transitifs statifs de relation repère-thème sans diathèses (sous-types contenir, regorger de)}

Les verbes statifs de relation repère-thème à voix active stative sont des prédicats de localisation interne transitifs, les uns transitifs directs, les autres transitifs indirects, et sans diathèses.

\subsection{Sous-type contenir/receler : voix active stative non passivable}

Les verbes de localisation interne contenir et receler sont des verbes transitifs directs non passivables : ce musée (contient, recèle) des chefs-d'oeuvre inattendus vs * des chefs-d'oeuvre inattendus sont (contenus, recelés) par ce musée. Ils n'ont pas davantage de diathèse de réduction du complément : * Ce musée (contient, recèle).

\subsection{Sous-type regorger de/déborder de : voix active stative en de}

Les verbes de localisation interne déborder de et regorger de sont des verbes transitifs indirects sans diathèses : ce musée (déborde, regorge) de chefs-d'oeuvre inattendus. Ils n'ont pas davantage de diathèse de réduction du complément: ${ }^{*}$ Ce musée (déborde, regorge).

\section{$5 \quad$ Verbes de relation repère-thème à voix passive stative sans voix active correspondante (sous-types être bourré de, gorgé de)}

Les verbes de relation repère-thème à voix passive stative n'ont pas de voix active correspondante. En revanche, ils admettent une diathèse d'augmentation du nombre des arguments, puisqu'on peut les conjuguer à la voix active et à la voix passive causatives. Leur schéma de diathèses est donc le suivant :

(12)-1 Absence de diathèse de permutation faisant alterner la voix passive non causative stative avec la voix active non causative stative : 
* Depuis deux jours, de l'eau (gorge, gorgeait) le sol

(12)-2 Diathèse d'augmentation du nombre des arguments faisant alterner la voix passive non causative stative avec la voix active causative télique :

Depuis deux jours, le sol (est, était) gorgé d'eau (cf. (12)-1)

En deux minutes, la pluie (gorgea, gorgera) le sol d'eau

Depuis deux minutes, la pluie (gorge, gorgeait) le sol d'eau

(12)-3 Diathèse de permutation faisant alterner la voix active causative télique avec la voix passive causative télique :

En deux minutes, la pluie (gorgea, gorgera) le sol d'eau (cf. (12-2))

En deux minutes, le sol (fut, sera) gorgé d'eau par la pluie

Depuis deux minutes, la cour (est, était) gorgé d'eau par la pluie

Certains verbes de cette catégorie (gorger, napper) ont en outre une diathèse donnant lieu à une voix médio-passive télique :

(12)-4 Depuis deux jours, le sol (est, était) gorgé d'eau (cf. (12)-1)

En deux minutes, le sol se (gorgea, gorgera) d'eau

\subsection{Sous-type être bourré de : voix passive stative en de \& causatif (actif et passif téliques)}

\section{- Verbes de localisation interne}

Les verbes bourrer de, fourrer de localisent des objets massifs ou pluriels. Le verbe truffer de localise des objets pluriel. Les verbes additionner de et étendre de localisent un liquide dans un autre liquide.

\section{- Verbes de localisation externe avec contact}

Les verbes enduire, oindre et tartiner (familier) localisent un objet massif semi-liquide sur une surface. Le verbe mouiller localise un liquide sur un solide. Les verbes asperger et éclabousser localisent un objet liquide ou semi-liquide « atomisé » sur une surface. Le verbe saupoudrer localise un certain type d'objet massif sur une surface. Le verbe inonder localise un liquide sur une surface, avec un effet holistique. Il a la particularité d'avoir une diathèse de réduction de la variante passive stative :

$$
\begin{aligned}
& \text { Le jardin est inondé d'eau de pluie } \\
& \text { Le jardin est inondé }
\end{aligned}
$$

\subsection{Sous-type être gorgé de : voix passive stative en de, voix médio-passive télique \& causatif (actif et passif téliques non ponctuels)}

- Verbe de localisation interne : gorger de (cf. ci-dessus exemples (12)-1 à (12)-4)

- Verbe de localisation externe : napper de, qui localise un objet massif semi-liquide sur une surface 


\section{Les verbes combinant des diathèses à complément d'objet avec des diathèses à complément prépositionnel locatif}

Les verbes de la catégorie qui va être étudiée combinent deux types de structure argumentale : une structure transitive directe ou indirecte et une structure à argument prépositionnel locatif. A propos de ce type de situation, B. Levin \& M. Rappaport Hovav 2005: 186-236 parlent de multiple argument realization, phénomène qui se manifeste notamment dans les locative alternations, par exemple la Spray/load Alternation (B. Levin 1993) ou la Swarm Alternation (M. Salkoff 1983).

\subsection{Verbes transitifs directs à voix active stative et à voix causative prépositionnelle locative (sous-type inclure)}

(13) Diathèse d'augmentation faisant alterner la voix active non causative stative avec la voix active causative télique ponctuelle (achievement) à argument prépositionnel locatif :

\section{L'enveloppe inclut un chèque \\ J'ai inclus un chèque dans l'enveloppe}

Les verbes transitifs directs concernés sont de localisation interne pour les verbes renfermer, englober et inclure. Les verbes cacher et dissimuler sont de localisation interne ou externe : Le (paravent, coffre) dissimule des tableaux vs dissimuler des tableaux dans un coffre, dissimuler des tableaux derrière un paravent.

\subsection{Verbes transitifs indirects à voix active non causative stative, et à voix active non causative stative à argument locatif (sous-types abonder en et grouiller de)}

Ces diathèses (cf. Boons, Guillet, Leclère 1976) sont ici non plus d'augmentation, mais de permutation.

(14)-1 Diathèse de permutation faisant alterner la voix active non causative stative à complément en en, avec la voix active non causative stative à argument prépositionnel locatif :

Cette région abonde en eau

L'eau abonde dans cette région

Les verbes concernés sont abonder et surabonder.

(14)-2 Diathèse de permutation faisant alterner la voix active non causative stative à complément en de avec la voix active non causative stative à argument prépositionnel locatif :

Cette région pullule de moustiques

Les moustiques pullulent dans cette région

Les verbes concernés sont foisonner, fourmiller, grouiller, pulluler, vibrionner.

\subsection{Verbes à voix passive stative en de et à voix causative prépositionnelle locative (sous-type charger)}

Cette catégorie, beaucoup plus petite en français qu'en anglais, correspond à peu près à celle des Spray/Load verbs de B. Levin 1987: 50-51.

(15)-1 Diathèse d'augmentation du nombre des arguments faisant alterner la voix passive non causative stative avec la voix active causative télique : 


\section{La plate-forme du camion est chargée de feuilles mortes}

Pierre a chargé la plate-forme du camion de feuilles mortes : sujet agentif (animé)

Le vent a chargé la plate-forme du camion de feuilles mortes : sujet causal (prédicatif)

(15)-2 Diathèse d'augmentation faisant alterner la voix médio-passive télique avec la voix active causative télique (avec argument sujet causal) :

La plate-forme du camion s'est chargée de feuilles mortes

Le vent a chargé la plate-forme du camion de feuilles mortes (sujet causal) ; cf. (15)-1

(15)-3 Diathèse de permutation des arguments repère et thème faisant alterner la voix active causative télique à sujet agentif avec la voix active causative (télique) à argument prépositionnel locatif

Pierre a chargé la plate-forme du camion de feuilles mortes (sujet agentif) ; cf. (15)-1

Pierre a chargé des feuilles mortes sur la plate-forme du camion

(15)-4 Diathèse de permutation des arguments agent et thème faisant alterner la voix active causative (télique) à argument locatif avec la voix passive télique à argument locatif :

Pierre a chargé des feuilles mortes sur la plate-forme du camion (cf. (15)-3)

Des feuilles mortes ont été chargées par Pierre sur la plate-forme du camion

Le verbe charger est le seul à pouvoir être conjugué à la voix moyenne. Les verbes barbouiller et peinturlurer ainsi que les verbes semer, piquer et planter ne disposent pas de la voix moyenne. Ce phénomène est lié au fait que ces verbes sélectionnent des noms animés en position d'argument causatif.

\subsection{Les sous-types baigner et envelopper}

Le sous-type baigner présente une riche combinaison de diathèses transitives directes et indirectes, et de diathèses à argument locatif :
Un brouillard doré baigne les arbres
Les arbres sont baignés d'un brouillard doré
L'aurore baigne les arbres d'un brouillard doré
Les arbres baignent dans un brouillard doré
L'aurore baigne les arbres dans un brouillard doré
Les arbres sont baignés par l'aurore dans un brouillard doré

Le sous-type envelopper se distingue du précédent par le fait de ne pas disposer, au sein des diathèses locatives, de la voix active stative :
Un brouillard doré enveloppe les arbres
Les arbres sont enveloppés d'un brouillard doré
L'aurore enveloppe les arbres d'un brouillard doré
* Les arbres enveloppent dans un brouillard doré
L'aurore enveloppe les arbres dans un brouillard doré
Les arbres sont enveloppés par l'aurore dans un brouillard doré

\section{Conclusion}

Nous avons voulu dans cet article souligner l'importance primordiale, dans le travail de classification syntactico-sémantique des verbes, des diathèses et des modes d'action qui s'y manifestent. 
La plupart des verbes transitifs de localisation statique permettent la linéarisation des arguments dans l'ordre repère de la localisation / thème de la localisation, alors que tous les verbes de localisation à arguments prépositionnels locatifs les linéarisent dans l'ordre inverse. On peut en déduire que la «fonction» principale des verbes transitifs est d'offrir aux locuteurs la possibilité de topicaliser l'argument repère de la localisation. Concernant les verbes transitifs directs de localisation linéarisant les arguments dans l'ordre thème/repère (cf. supra Section 3), on admet assez généralement, depuis S.R. Anderson 1971, que leur fonction est de permettre un effet holistique qui n'existe pas dans le vocabulaire des verbes de localisation statique à argument prépositionnel. Cette hypothèse n'est pas vérifiée dans la totalité des cas. Une étude plus approfondie de la catégorie qui fait l'objet de cet article devrait permettre d'en savoir plus sur ce qui conditionne l'effet holistique.

\section{Notes}

1. Il n'est pas question dans cet article des prédicats incorporant dans leur base le radical d'un nom de tache, de marque ou de déformation (ex. Deux balafres sanguinolentes balafraient le visage de Pierre vs Le visage de Pierre était balafré de deux balafres sanguinolentes). Nous ne croyons pas qu'ils expriment une relation de localisation, car ces noms sont des méronymes des noms de surface. Or les relations méronyme/holonyme ne sont pas selon nous des relations de localisation (cf. infra note 2). Quant aux noms d'ingrédients qui incorporent également leur radical dans la base de certains verbes (ex. huiler, saler, phosphater, oxygéner), mais qui ne sont pas des méronymes, il n'en est pas davantage question, car rien ne prouve que la relation d'appartenance qu'expriment de tels verbes soit d'essence locative. Un étude approfondie des 3174 entrées de la Classe N (Verbes de munir/démunir) de Dubois \& DuboisCharlier 1997 devrait aider à résoudre ce problème. Pour une opinion opposée à la nôtre, cf. A. Guillet et C. Leclère 1992 et M. Labelle 1992, qui ont beaucoup étudié ces verbes.

2. Pour une opinion contraire, voir F. Martin 2006:523. L'argument selon lequel, dans la phrase La place était baignée d'ombres par les platanes, le segment «d'ombres » ne saurait être un complément d'agent parce que la phrase comporte déjà le complément d'agent "par les platanes» fait abstraction du fait que le verbe baigner figure là dans son emploi causatif, si bien que la phrase comporte deux prédications sous-jacentes enchâssées l'une dans l'autre : les platanes FAISAIENT (des ombres BAIGNER la place). La thèse de F. Martin est que «le de-GP des verbes locatifs n'est pas une variante du complément d'agent en par, mais un complément génitif de matière ou de partie » (p. 521). On pourrait objecter que le fait d'être un génitif n'est pas en soi contradictoire avec le fait d'être un complément d'agent. Mais l'essentiel est de dire que, si des verbes à complément de forme $d e-G P$ étaient des verbes de relation matière/forme ou de relation partie/tout, alors il ne faudrait pas en déduire que ce sont des verbes locatifs, car ces relations n'impliquent pas nécessairement l'existence d'une relation de localisation. En particulier, la relation méronyme/holonyme doit être soigneusement distinguée de la relation objet à localiser/point de repère, non seulement à cause leur extrême différence sémantique, mais aussi parce qu'il est fréquent que la méréologie exclue la localisation, par exemple dans le vocabulaire des méronymes de partie fonctionnelle (les murs d'une maison ne sont localisés ni dans la maison, ni sur la maison, ni à l'extérieur de la maison). Sur les propriétés linguistiques de quelques classes de méronymes, voir Le Pesant 2000.

\section{Références}

Anderson, S.R.1971. “On the Role of Deep Structure in Semantic Interpretation”. Foundations of Language 7.

Aurnague, M. 2004. Les structures de l'espace linguistique. Louvain-Paris : Peeters.

Boons, J.-P., A. Guillet \& C. Leclère. 1976. La structure des phrases simples en français. I. Constructions intransitives. Genève-Paris : Droz.

Borillo, A. 1998. L'espace et son expression en français. Gap-Paris : Ophrys.

Dubois, J. \& F. Dubois-Charlier. 1997. Les verbes français. Paris : Larousse.

Guillet, A. \& C. Leclère. 1992. La structure des phrases simples en français. Constructions transitives locatives. Genève-Paris : Droz. 
DOI $10.1051 / \mathrm{cmlf0} 8068$

Labelle, M. 1992. « La structure argumentale des verbes locatifs à base nominale ». Lingvisticae Investigationes, vol. 16/2, 267-315. Amsqterdam : John Benjamins.

Le Pesant, D. 2000. « Suggestions méthodologiques pour une typologie des classes de méronymes », in Choi-Jonin, Gerhard-Krait \& Riegel éds. Actes de la journée «Les relations partie-tout ». Scolia 12:161-179. Strasbourg: Publications de l'Université Marc Bloch.

Levin, B. 1993. English Verb Classes and Alternations. Chicago : University of Chicago Press.

Levin, B. \& M. Rappaport Hovav. 2005. Argument Realization. Cambridge : University Press.

Martin, F. 2006. «De N-nus indéfinis prédicatifs et prédicats incorporants », in Corblin, Ferrando, Kupferman, éds. Indéfini et Prédication. Paris : PUPS. 521-534.

Salkoff, M. 1983. "Bees are Swarming in the Garden”. Language 53: 288-346.

Vandeloise, C. 1986. L'espace en français. Paris : Le Seuil.

Vendler, Z. 1967. Linguistics in Philosophy. Ithaca : Cornell University Press. 